\title{
Reversible Pre-Capillary Pulmonary Hypertension Due to Dasatinib
}

\author{
Herminia L Buchelli Ramirez MD, Celso M Álvarez Álvarez MD, José J Rodríguez Reguero PhD, \\ Marta M García Clemente $\mathrm{PhD}$, and Pere Casan Clarà PhD
}

\begin{abstract}
Pulmonary arterial hypertension and secondary pleural effusion have been reported in association with long-term therapy with the multi-tyrosine kinase inhibitor dasatinib, approved for the treatment of chronic myeloid leukemia. Here, we present the case of a 50-year-old man, diagnosed with chronic myeloid leukemia in August 2003, who developed pulmonary arterial hypertension after $>4$ years of treatment with dasatinib. The complete remission of pulmonary arterial hypertension following dasatinib discontinuation suggests an etiological role of the drug in its development, although the administration of sildenafil may have played a therapeutic role. Key words: pulmonary hypertension; chronic myeloid leukemia; long-term therapy; drug-induced. [Respir Care 2014;59(5):e77-e80. (C) 2014 Daedalus Enterprises]
\end{abstract}

\section{Introduction}

Pulmonary arterial hypertension is defined as a group of diseases characterized by a mean pulmonary artery pressure $\geq 25 \mathrm{~mm} \mathrm{Hg}$ and a progressive increase in pulmonary vascular resistance leading to right ventricle failure. The proposed causes of pulmonary arterial hypertension range widely, and one of these may be associated with the use of drugs. ${ }^{1,2}$

Dasatinib is a tyrosine kinase inhibitor (TKI) that also inhibits platelet-derived growth factor (PDGF) receptor B, Src, and c-Kit. It is approved for use in patients with chronic myeloid leukemia (CML) who have developed resistance, suboptimal response, or intolerance to imatinib. ${ }^{3}$

Pulmonary arterial hypertension in conjunction with pleural effusion secondary to dasatinib has been described in a few patients. This phenomenon was observed in patients after long-term treatment with dasatinib. ${ }^{4,5}$ We pre-

Drs Buchelli Ramierez, Álvarez Álvarez, Rodríguez Reguero, García Clemente, and Casan Clarà are affiliated with the Pneumology Department, and Dr Reguero is affiliated with the Cardiology Department Asturias Central University Hospital, Oviedo, Asturias, Spain.

The authors have declared no conflicts of interests.

Correspondence: Herminia L Buchelli Ramirez MD, Pneumology Department, Asturias Central University Hospital, Calle del Doctor Bellmunt S/N Oviedo, Asturias 33006, Spain. E-mail: hlbuchellir@gmail.com.

DOI: $10.4187 /$ respcare.02692 sent the following case of severe pulmonary arterial hypertension associated with the use of this drug.

\section{Case Report}

In August 2003, a 50-y-old man who was an ex-smoker (20 pack-years) was diagnosed with CML (BCR/ABL-positive in chronic phase). He began treatment in October 2003 with imatinib, initially at a dose of $400 \mathrm{mg} / \mathrm{d}$ and subsequently increased to $800 \mathrm{mg} / \mathrm{d}$, with a good response. In June 2007, owing to the persistent loss of major molecular remission, this treatment was suspended and replaced by another TKI (dasatinib at $100 \mathrm{mg} / \mathrm{d}$ ). The patient began to suffer a range of symptoms, including cramps, isolated episodes of diarrhea, somnolence, and arthralgia, which caused problems in his working life but were considered insufficient to justify treatment interruption.

In June 2011, he was admitted to the respiratory disease ward with a clinical history of dyspnea, which had progressively worsened over a period of 2 months, with repeated episodes of thoracic pain related to effort and accompanied by a hacking cough and wheezing sounds on auscultation. His physician prescribed bronchodilator treatment, with no improvement in his condition. The results of physical examination were: blood pressure, $120 / 80 \mathrm{~mm} \mathrm{Hg}$; temperature, $36^{\circ} \mathrm{C}$; breathing frequency, 22 breaths/min; and heart rate, 83 beats/min. Auscultation showed absence of normal breath sounds up to two thirds of the right hemithorax. Heart sounds, abdominal examination, and extremities were all normal. Laboratory tests for glucose, 

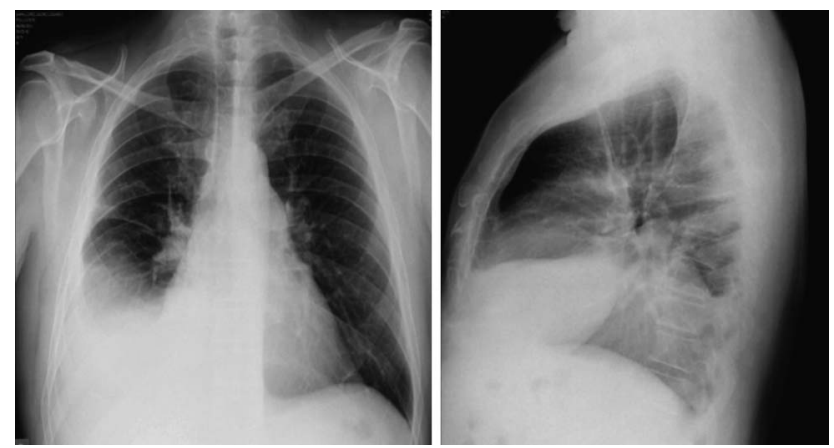

Fig. 1. Chest $x$-ray posteroanterior and lateral views: right pleural effusion with atelectasis. In-circuit test limit.

urea, creatinine, ions, hepatic and lipid profiles, human immunodeficiency virus (HIV) serology, and antinuclear antibody and anti-neutrophil cytoplasmic antibody serology were all normal. An electrocardiogram showed sinus rhythm with right axis deviation and negative $\mathrm{T}$ waves in the right precordial leads. Chest x-ray showed right pleural effusion (Fig. 1). Pleural fluid from a diagnostic thoracentesis was compatible with lymphocytic exudate, negative on culture, with no flow cytometry evidence of hematolymphoid infiltration and no cytological evidence of malignancy. Pulmonary function tests showed normal spirometry results and a reduction in diffusing capacity of the lung for carbon monoxide $\left(\mathrm{D}_{\mathrm{LCO}}\right)$. On computed tomography $(\mathrm{CT})$ angiogram, an increase in the right atrium and ventricle was noted. The absence of perfusion alterations in the pulmonary arteries allowed pulmonary thromboembolism to be ruled out. Scintigraphy ventilation/perfusion lung scan showed hypoventilation and hypoperfusion of the right lung, a finding related to the presence of pleural effusion. There were no peripheral perfusion alterations suggestive of residual lesions after previous pulmonary thromboembolism. Abdominal ultrasound did not identify signs suggesting portal hypertension.

The level of amino-terminal pro-brain natriuretic peptide (NT-proBNP) was $7312 \mathrm{pg} / \mathrm{L}$, and the result for the 6-min walk test (6MWT) was $420 \mathrm{~m}$. The results of a transthoracic echocardiogram and right heart catheterization are shown in Table 1. Taking into consideration all of these data, a diagnosis of severe pulmonary hypertension attributable to dasatinib was reached. During hospitalization, treatment with dasatinib was suspended, and the patient began treatment with sildenafil at $20 \mathrm{mg} / 8 \mathrm{~h}$. Eleven days after the suspension of dasatinib, the pleural effusion had disappeared. In September 2011, treatment with second-generation TKIs (nilotinib at $600 \mathrm{mg} / \mathrm{d}$ ) for his CML was added, and the dose of sildenafil was increased to $40 \mathrm{mg} / 8 \mathrm{~h}$.

During the first 2 months after discharge, echocardiographic signs of right ventricular failure persisted. At 6 months, there was clear improvement in clinical symp-
Table 1. Right Heart Catheterization, Transthoracic

Echocardiography, 6-Minute Walk Test, and AminoTerminal Pro-Brain Natriuretic Peptide Parameters at Baseline and After Dasatinib Discontinuation

\begin{tabular}{|c|c|c|c|}
\hline & $\begin{array}{l}\text { June } \\
2011\end{array}$ & $\begin{array}{l}\text { August } \\
2012\end{array}$ & $\begin{array}{c}\text { March } \\
2013\end{array}$ \\
\hline WHO/NYHA FC & IV & II & I \\
\hline \multicolumn{4}{|l|}{ Right heart catheterization } \\
\hline $\begin{array}{l}\text { Right ventricular systolic pressure } \\
\qquad(\mathrm{mm} \mathrm{Hg})\end{array}$ & 82 & 36 & 44 \\
\hline $\begin{array}{l}\text { Pulmonary artery pressure mean } \\
(\mathrm{mm} \mathrm{Hg})\end{array}$ & 52 & 19 & 20 \\
\hline PVR (mm Hg·min/L) & 16 & 2 & 1 \\
\hline $\begin{array}{l}\text { Pulmonary-capillary wedge pressure } \\
(\mathrm{mm} \mathrm{Hg})\end{array}$ & 11 & 3 & 12 \\
\hline Right atrial pressure $(\mathrm{mm} \mathrm{Hg})$ & 10 & 2 & 2 \\
\hline $\mathrm{CO}(\mathrm{L} / \mathrm{min})$ & 2.6 & 6.7 & 6.1 \\
\hline Cardiac index $\left(\mathrm{L} / \mathrm{min} / \mathrm{m}^{2}\right)$ & 1.2 & 3.2 & 2.9 \\
\hline \multicolumn{4}{|l|}{ Transthoracic echocardiography } \\
\hline $\begin{array}{l}\text { Pulmonary artery systolic pressure } \\
(\mathrm{mm} \mathrm{Hg})\end{array}$ & 60 & 34 & NA \\
\hline TAPSE $(\mathrm{mm})$ & 16 & 24 & NA \\
\hline Systolic function & Reduced & Normal & NA \\
\hline 6MWT (m) & 420 & 540 & 480 \\
\hline NT-proBNP (pg/L) & 7312 & 28 & 80 \\
\hline
\end{tabular}

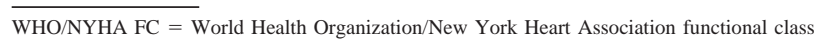
$\mathrm{PVR}=$ pulmonary vascular resistance

$\mathrm{CO}=$ cardiac output

TAPSE $=$ tricuspid annular plane systolic excursion

$6 \mathrm{MWT}=6$-min walk test

NT-proBNP $=$ amino-terminal pro-brain natriuretic peptide

$\mathrm{NA}=$ not available

toms, NT-proBNP, $\mathrm{D}_{\mathrm{LCO}}$, echocardiograms, and 6MWT. One year after the diagnosis, normalization of all of the hemodynamic parameters was observed on right cardiac catheterization and echocardiography (see Table 1), and the dose of sildenafil was therefore reduced to $20 \mathrm{mg} / 8 \mathrm{~h}$. In the final follow-up at 21 months after diagnosis, the patient was stable, with results indicating good prognosis: NT-proBNP of $80 \mathrm{pg} / \mathrm{L}$ and New York Heart Association functional classes I and II. The final right heart catheterization performed in March 2013 (see Table 1) confirmed complete resolution of pulmonary arterial hypertension. Repeat pulmonary function testing after the resolution of pulmonary arterial hypertension showed increased $\mathrm{D}_{\mathrm{LCO}}$ from $63 \%$ to $91 \%$.

\section{Discussion}

Pulmonary hypertension is defined hemodynamically as a mean pulmonary artery pressure $>25 \mathrm{~mm} \mathrm{Hg} .{ }^{1}$ The first category of the clinical classification termed pulmonary arterial hypertension includes idiopathic pulmonary arterial hypertension; heritable pulmonary arterial hyperten- 


\section{Reversible Pre-Capillary Pulmonary Hypertension Due to Dasatinib}

sion; and pulmonary arterial hypertension related to risk factors or associated conditions, ${ }^{2}$ such as connective tissue diseases, congenital heart diseases, portal hypertension, HIV infection, and exposure to drugs and toxins. ${ }^{6}$

Dasatinib is a multi-TKI approved for first- and secondline therapy of CML.7 Accumulated evidence indicates that receptor tyrosine kinases (RTKs) play an important role in the pathogenesis of pulmonary arterial hypertension, and inhibition of some specific RTK signaling may represent an attractive option for treatment of the disease. A 24-week randomized, double-blind, placebo-controlled pilot study showed that imatinib was associated with significant hemodynamic improvement, although not with any significant change in the primary end point (6MWT). ${ }^{8}$

The association between pulmonary arterial hypertension and dasatinib is paradoxical because the drug also acts as a potent RTK inhibitor. In contrast to imatinib, dasatinib is a potent inhibitor of additional important families of RTKs, including Src and ephrin receptors/ephrin kinases. It may therefore be speculated that Src inhibition plays a role in the development of dasatinib-associated pulmonary arterial hypertension. It has recently been reported that dasatinib inhibits PDGF-induced proliferation and migration of vascular smooth muscle cells via inhibition of both PDGF receptor activity and PDGF-dependent Src activation downstream of the PDGF receptor, which would in theory be beneficial in pulmonary arterial hypertension. ${ }^{4,9}$

Severe cardiovascular and respiratory adverse effects are infrequent in CML patients treated with dasatinib at the recommended dose of $100 \mathrm{mg} / \mathrm{d}$. Pulmonary arterial hypertension and pleural effusion were not observed in patients enrolled in a phase- 3 clinical trial and assigned to receive dasatinib at $100 \mathrm{mg} / \mathrm{d}$. On the contrary, this dosage significantly minimized the occurrence of key adverse events. This regimen does not affect short- or long-term efficacy, and at these low doses, only a few cases of pulmonary arterial hypertension have been reported. ${ }^{3,10}$ However, the occurrence of late-onset symptomatic pulmonary arterial hypertension (48 mo after starting dasatinib in our case) may reflect a chronic pathological mechanism that, after an insidious onset, might become clinically important while on long-term therapy with TKIs. These drugs are typically lifelong therapies, ${ }^{5,7}$ and information on longterm adverse effects is therefore of considerable clinical interest. In the French pulmonary hypertension registry, the lowest estimate of incidence of pulmonary arterial hypertension in patients exposed to dasatinib was $0.45 \% .^{8}$

The incidence of pleural effusion in patients receiving dasatinib was 17\% (grades 3-4 in 4\%). In a recent report by Quintás-Cardama et al, ${ }^{11}$ pleural effusion occurred in $48(35 \%)$ of 138 patients treated with dasatinib. Most pleural effusions were exudative, arguing against primary car- diac impairment as the mechanism of pleural fluid accumulation. ${ }^{11,12}$

In our case, other conditions associated with pulmonary arterial hypertension, such as pulmonary embolism or parenchymal lung disease, were ruled out by chest CT scan with contrast, CT angiogram, and scintigraphy ventilation/ perfusion lung scan. Hepatic ultrasound excluded portopulmonary hypertension, and bubble contrast echocardiogram excluded congenital heart disease, which may be associated with pulmonary arterial hypertension. Furthermore, laboratory screening tests for HIV and systemic rheumatic diseases, including antinuclear antibodies, were negative. There was no clinical evidence of pulmonary arterial hypertension when dasatinib was initiated, and the echocardiogram performed (1 y before starting dasatinib) was normal. These findings support the diagnosis of pulmonary arterial hypertension associated with dasatinib.

Dasatinib was discontinued based on suspicion of its involvement in pulmonary arterial hypertension. During follow-up, we performed right heart catheterization on two occasions. At 1 year, it showed normalization of all hemodynamic parameters, which coincided with complete resolution of breathlessness, and the dose of sildenafil was therefore reduced to $20 \mathrm{mg} / 8 \mathrm{~h}$. At final follow-up 21 months after diagnosis, right heart catheterization showed no evidence of recurrent pulmonary arterial hypertension, but the targeted pulmonary arterial hypertension therapy with sildenafil was not discontinued until months later to avoid the possible consequences of abrupt discontinuation.

Concerning the role of nilotinib in resolving pulmonary arterial hypertension (initiated 1 month after diagnosis in our patient), we cannot affirm or deny its therapeutic value. Zakrzewski et al ${ }^{13}$ presented the first case report of pulmonary hypertension in a patient treated with nilotinib for CML. They found that the change in pulmonary artery pressure was dose-dependent and that the decrease occurred after discontinuation of nilotinib. However, a clinical trial is currently investigating the efficacy, safety, tolerability, and pharmacokinetics of nilotinib in pulmonary arterial hypertension, the results of which may help clarify its therapeutic usefulness.

In our patient, complete resolution of pulmonary arterial hypertension was noted several months after discontinuation of dasatinib and after the introduction of sildenafil therapy, with mean pulmonary artery pressure levels within the normal range to date. This is remarkable because complete remission and normalization of pulmonary hemodynamics are not usually achieved in pulmonary arterial hypertension by medical treatment. However, in our case, resolution was achieved not only by discontinuation of dasatinib but also after therapy with sildenafil. Similarly, in a case reported by Dumitrescu et al, ${ }^{4}$ the development of severe pulmonary arterial hypertension was associated with 


\section{Reversible Pre-Capillary Pulmonary Hypertension Due to Dasatinib}

dasatinib treatment for CML, which completely resolved after TKI withdrawal and the administration of targeted pulmonary arterial hypertension therapy (6 mo) with sildenafil.

In conclusion, we have reported here a case of severe pulmonary arterial hypertension fulfilling the criteria of drug-induced pulmonary arterial hypertension after complete invasive hemodynamic evaluation, which suggests a direct and specific effect of dasatinib on the pulmonary vasculature. Physicians need to be aware of this possible adverse effect of dasatinib to appropriately monitor and manage these patients.

\section{REFERENCES}

1. Task Force for Diagnosis and Treatment of Pulmonary Hypertension of European Society of Cardiology (ESC), European Respiratory Society (ERS), International Society of Heart and Lung Transplantation (ISHLT), Galiè N, Hoeper MM, Humbert M, et al. Guidelines for the diagnosis and treatment of pulmonary hypertension. Eur Respir J 2009;34(6):1219-1263.

2. Simonneau G, Robbins IM, Beghetti M, Channick RN, Delcroix M, Denton $\mathrm{CP}$ et al. Updated clinical classification of pulmonary hypertension. J Am Coll Cardiol 2009;54(1 Suppl):S43-S54.

3. Porkka K, Khoury HJ, Paquette RL, Matloub Y, Sinha R, Cortes JE. Dasatinib $100 \mathrm{mg}$ once daily minimizes the occurrence of pleural effusion in patients with chronic myeloid leukemia in chronic phase and efficacy is unaffected in patients who develop pleural effusion. Cancer 2010;116(2):377-386.

4. Dumitrescu D, Seck C, ten Freyhaus H, Gerhardt F, Erdmann E, Rosenkranz S. Fully reversible pulmonary arterial hypertension as- sociated with dasatinib treatment for chronic myeloid leukaemia. Eur Respir J 2011;38(1):218-220.

5. Orlandi EM, Rocca B, Pazzano AS, Ghio S. Reversible pulmonary arterial hypertension likely related to long-term, low dose dasatinib treatment for chronic myeloid leukaemia. Leuk Res 2012;36(1):e4e6.

6. Adir Y, Humbert M. Pulmonary hypertension in patients with chronic myeloproliferative disorders. Eur Respir J 2010;35(6):1396-1406.

7. Hennigs JK, Keller G, Baumann HJ, Honecker F, Kluge S, Bokemeyer $\mathrm{C}$, et al. Multi tyrosine kinase inhibitor dasatinib as novel cause of severe pre-capillary hypertension? BMC Pulm Med 2011; $11: 30$

8. Montani D, Bergot E, Günther S, Savale L, Bergeron A, Bourdin A, et al. Pulmonary arterial hypertension in patients treated by dasatinib. Circulation 2012;125(17):2128-2137.

9. Chen Z, Lee FY, Bhalla KN, Wu J. Potent inhibition of plateletderived growth factor-induced responses in vascular smooth muscle cells by BMS-354825 (dasatinib). Mol Pharmacol 2006;69(5):15271533.

10. Shah NP, Kantarjian HM, Kim DW, Réa D, Dorlhiac-Llacer PE, Milone JH, et al. Intermittent target inhibition with dasatinib $100 \mathrm{mg}$ once daily preserves efficacy and improves tolerability in imatinibresistant and -intolerant chronic-phase chronic myeloid leukemia. J Clin Oncol 2008;26(19):3204-3212.

11. Quintás-Cardama A, Kantarjian H, O’Brien S, Borthakur G, Bruzzi J, Munden R, Cortes J. Pleural effusion in patients with chronic myelogenous leukemia treated with dasatinib after imatinib failure. J Clin Oncol 2007;25(25):3908-3914.

12. Rasheed W, Flaim B, Seymour JF. Reversible severe pulmonary hypertension secondary to dasatinib in a patient with chronic myeloid leukemia. Leuk Res 2009;33(6):861-864.

13. Zakrzewski D, Seferynska I, Warzocha K, Hryniewiecki T. Elevation of pulmonary artery pressure as a complication of nilotinib therapy for chronic myeloid leukemia. Int J Hematol 2012;96(1): 132-135. 